\title{
Optimization of Bioreactor Volumes in Steady State - A Simulation Study
}

\author{
Hanna Molin $^{1} \quad$ Jesús Zambrano $^{2, *}$ Bengt Carlsson $^{1}$ \\ ${ }^{1}$ Department of Information Technology, Uppsala University, Uppsala, Sweden. \\ ${ }^{2}$ School of Business, Society and Engineering, Mälardalen University, Västerås, Sweden. \\ *Corresponding author e-mail: jesus.zambrano@mdh. se
}

\begin{abstract}
This work deals with the analysis and design of bioreactors formed by a number of completely stirred tank reactors (CSTRs) in series. The model includes three main components: the concentration of one dominant particulate biomass, one soluble substrate component and one particulate inert matter. The model is analyzed in steadystate conditions. Monod kinetics is used for describing the specific growth rate, and the decay rate of the biomass is included. Two main optimization problems were studied: (i) minimize the effluent substrate concentration for a given total volume, and (ii) minimize the total volume for a given effluent substrate concentration. An alternative to optimize a large number of CSTRs is to consider the asymptotic case of one CSTR followed by a plug-flow reactor (PFR). Numerical results show that there is an optimal volume distribution for the two configurations in each optimization problem.
\end{abstract}

Keywords: Bioreactor optimization, completely stirred tank, effluent minimization, plug-flow reactor.

\section{Introduction}

Modeling and analysis of bioreactors in wastewater treatment processes have been active research areas in the last 50 years, see Grady Jr. et al. (1999) and the references therein. Special attention has been placed in the steadystate analysis because it can provide guidelines for achieving a proper process operation and optimization. During those decades, the optimization of bioreactors has also been extensively analyzed. Every new contribution aims to design methods for analyzing and optimizing the volume distribution of completely stirred tank reactors (CSTRs) in series.

In general, the aim is to design the process in such a way that the substrate concentration of a certain incoming flow can be minimized or reduced, even by using one or more bioreactors in series (de Gooijer et al., 1996). Hence, the optimization of CSTRs usually involves finding the optimal distribution of volumes for a given effluent substrate concentration, see Abu-Reesh (1996).

Alternatives to solve the optimization of CSTRs includes graphical solutions referred as nomograms, which give an approximate graphical computation of mathemat- ical functions (Braha and Hafner, 1985).

Most of the analysis and optimization of CSTRs assumes up to two main components in the model: dissolved substrate and particulate biomass. Some examples are mentioned as follows. Asymptotic solutions for the effluent concentration leaving a system of up to $N=4$ CSTRs in series were presented by Nelson and Holder (2009). The analysis assumes equally-sized CSTRs and Contois kinetics (Contois, 1959) for the biomass growth rate. Two scenarios were studied, when a total residence time $\left(\tau_{t}\right)$ is given, and when this residence time is slightly larger than the wash-out condition. In the first scenario, results show that the effluent decreases with the form $1 / \tau_{t}^{N}$. In the second scenario, results show that a large decrease in the effluent concentration can be obtained by a small increase in $\tau_{t}$.

The optimal design of CSTRs in series has often been compared to the design of a plug-flow reactor (PFR). Another aspects is that, in practice, the behavior of a biological reactor is between a CSTR and a PFR (Diehl et al., 2016, 2017). The work by Luyben and Tramper (1982) presents an analytical expression for the minimum volume to achieve a certain effluent substrate concentration in a process with Monod kinetics. The work shows some numerical results for up to $N=10$ CSTRs in series considering two main scenarios: optimal volume distribution and equally sized volume. The case of a PFR was used for comparison. Results show that the difference between the first two volumes is larger for low $N$ and decreases as $N$ increases. Results also show that the case of $N$ CSTRs approaches to the case of a PFR as $N$ increases. Hill and Robinson (1989) derived an expression for different growth kinetics that could be used to find the minimum possible total residence time to obtain a certain substrate conversion for $N$ CSTRs. An illustration for Monod kinetics was included. The results were compared with a PFR and showed that for most cases, three optimally designed CSTRs in series give the same total mean residence time as a PFR. Abu-Reesh (1996) calculates the optimal design of $N$ CSTRs to get the minimum overall volume needed for a certain substrate conversion, and compares the results with the behavior of a PFR. The study used a Michaelis-Menten kinetics.

Zambrano et al. (2015) shows an approach for the opti- 
mal design of CSTRs in series when the number of CSTRs is large. The process assumes Monod kinetics for the biomass growth, and the model includes two main components: one particulate biomass and one soluble substrate. The biomass decay is negligible. Analytical expressions were derived for solving the following two scenarios: to find the optimal volume distribution for a given total volume so that the effluent substrate concentration can be minimized, and to find the minimum total volume that gives a certain effluent substrate concentration.

Recently, Gómez-Pérez and Espinosa (2017) analyzed the design of continuous bioreactors in series with recirculation. The bioreactors were represented by a system of linear equations. The growth rate was assumed to be constant and the decay of biomass was neglected. Nontrivial solutions to the equation system was found by using Singular Value Decomposition (SVD) as an analysis tool. The SVD analysis makes it possible to characterize the solutions to the system of equations, and thereby improve the design of bioreactors in series.

In the present work, we deal with a simulation analysis of the process presented in Zambrano et al. (2015). In this case, the inert particulate matter is included in the bioreactor model, as well as the decay rate of the biomass. Two optimization problems were investigated: (i) the optimal volume distribution of $N$ CSTRs in series in a given total volume in order to minimize a given effluent substrate concentration, and (ii) the minimum total volume needed for a given effluent substrate concentration. The optimization results were compared with the case of a process formed by a CSTR followed by a PFR.

\section{Materials and Methods}

\subsection{The bioreactor model}

Consider a bioreactor of volume $V_{\text {tot }}$ formed by $N$ CSTRs in series as shown in Figure 1, where each CSTR is modeled assuming one limiting substrate, one biomass and one inert particulate matter. The time-dependent ordinary

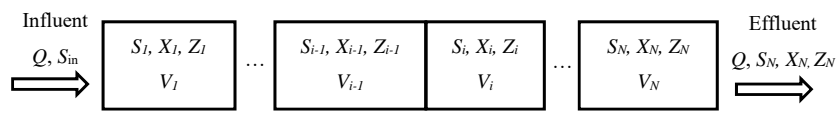

Figure 1. $N$ CSTRs in series.

differential equations that describe the dynamics of these three components in the $i$-th $(i=1,2, \ldots, N)$ CSTR are

$$
\begin{aligned}
\frac{\mathrm{d} S_{i}}{\mathrm{~d} t} & =-\left[\frac{\mu\left(S_{i}\right)}{Y}-\left(1-f_{p}\right) b\right] X_{i}+\frac{Q}{V_{i}}\left(S_{i-1}-S_{i}\right), \\
\frac{\mathrm{d} X_{i}}{\mathrm{~d} t} & =\left[\mu\left(S_{i}\right)-b\right] X_{i}+\frac{Q}{V_{i}}\left(X_{i-1}-X_{i}\right), \\
\frac{\mathrm{d} Z_{i}}{\mathrm{~d} t} & =f_{p} b X_{i}+\frac{Q}{V_{i}}\left(Z_{i-1}-Z_{i}\right),
\end{aligned}
$$

where the $i$-th CSTR has volume $V_{i}$, substrate concentration $S_{i}$, biomass concentration $X_{i}$ and inert concentration

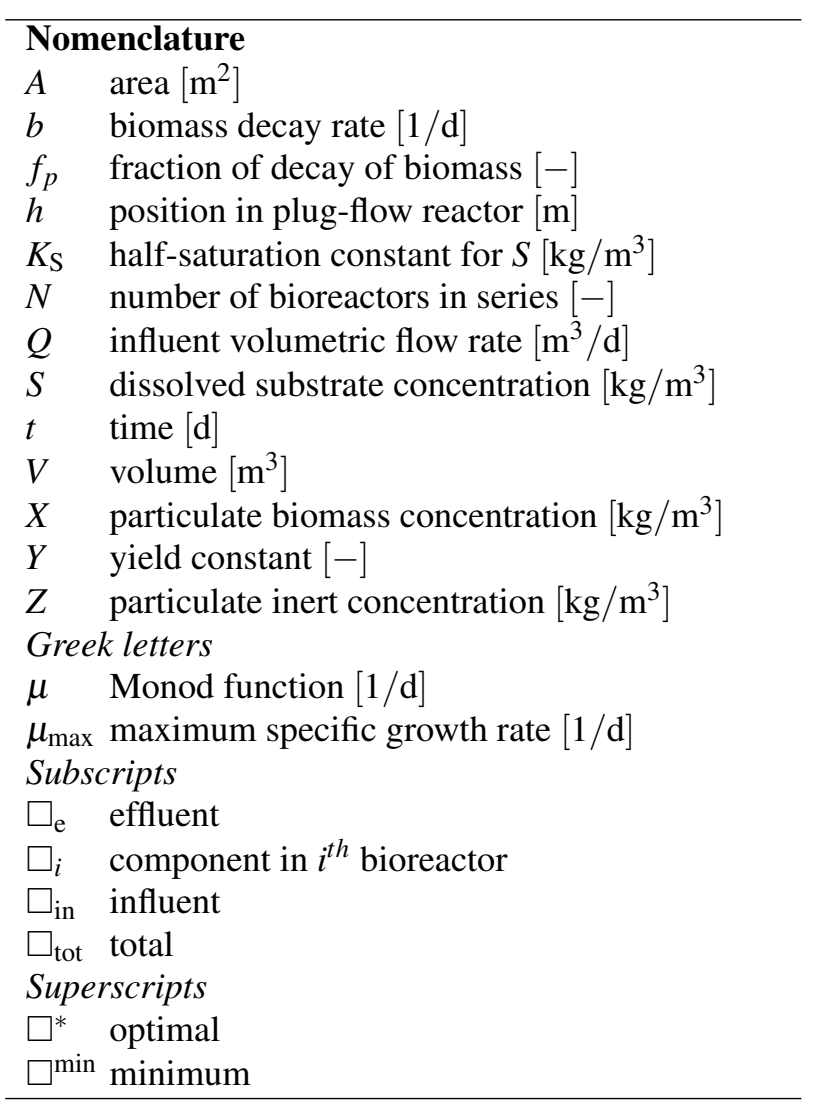

$Z_{i} . Y$ is the yield factor, $b$ is the decay rate of the biomass and $f_{p}$ is the fraction of decay of biomass $\left(0 \leq f_{p} \leq 1\right)$. The growth of the biomass is assumed to follow a single growth rate equation described by the Monod kinetics (Monod, 1949) given by

$$
\mu\left(S_{i}\right)=\mu_{\max } \frac{S_{i}}{K_{\mathrm{S}}+S_{i}},
$$

where $\mu_{\max }$ is the maximum specific growth rate, and $K_{\mathrm{S}}$ is a half-saturation constant. The influent and effluent volumetric flow rate are equal to $Q$.

The influent to the process is assumed to be formed only by a substrate concentration $S_{\text {in }}$, no biomass and inert concentration are present. Hence, from (1)-(3) and Figure 1 we have $S_{0}=S_{\text {in }}, X_{0}=X_{\text {in }}=0$ and $Z_{0}=Z_{\text {in }}=0$.

See in (1)-(3) that a fraction $f_{p}$ of the decay of biomass concentration is assumed to be inert concentration, whereas the remaining fraction $\left(1-f_{p}\right)$ is used as substrate.

\subsection{The steady-state expressions for CSTRs}

By assuming steady-state conditions in (1)-(3), an expression for $S_{i}$ can be obtained

$$
S_{i}=S_{i-1}-\frac{1}{Y}\left(X_{i}-X_{i-1}\right)-\frac{b}{Q Y}\left(1-\left(1-f_{p}\right) Y\right) V_{i} X_{i}
$$

For $N$ CSTRs in series, the recursive expression (5) gives

$$
S_{N}=S_{\text {in }}-\frac{1}{Y} X_{N}-\frac{b}{Q Y}\left(1-\left(1-f_{p}\right) Y\right) \sum_{n=1}^{N} V_{n} X_{n} .
$$


For a single CSTR, the solution for the substrate, biomass and inert concentration, and the minimum volume $\left(V_{1}^{\mathrm{min}}\right)$ to avoid wash-out are given by the following expressions

$$
\begin{aligned}
\mu\left(S_{1}\right) & =\frac{Q}{V_{1}}+b, \\
X_{1} & =\frac{Y Q\left(S_{\text {in }}-S_{1}\right)}{Q+V_{1} b\left(1-Y\left(1-f_{p}\right)\right)}, \\
Z_{1} & =\frac{f_{p} b V_{1} X_{1}}{Q}, \\
V_{1} & >V_{1}^{\min }=\frac{Q}{\mu\left(S_{\text {in }}\right)-b} .
\end{aligned}
$$

\subsection{The steady-state expression for a PFR}

When the number of $N$ CSTRs in series becomes large, the resulting process can be seen as a CSTR followed by a PFR. The CSTR is assumed to be large enough to avoid wash-out condition (i.e., $V_{1}>V_{1}^{\mathrm{min}}$ ). The PFR is formed by dividing the remaining volume $\left(V_{\text {tot }}-V_{1}\right)$ into a large number of volumes $\Delta V$, see Figure 2 .

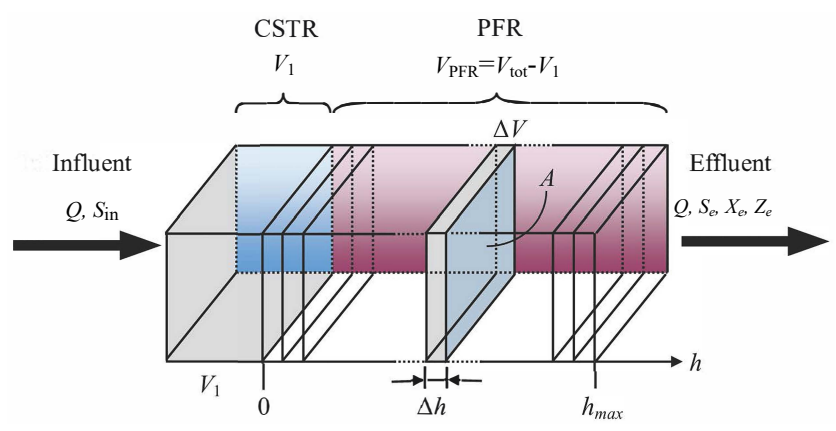

Figure 2. Dividing the remaining volume $\left(V_{\text {tot }}-V_{1}\right)$ into a large number of volumes $\Delta V$.

It is also assumed that each sliced bioreactor has the same area $A$, giving $\Delta V=A \Delta h$. For a small interval $(h, h+$ $\Delta h)$ the conservation of mass for the dissolved substrate gives

$$
\begin{gathered}
\underbrace{\frac{\mathrm{d}}{\mathrm{d} t} \int_{h}^{h+\Delta h} A S(x, t) \mathrm{d} x}_{\text {mass increase per time unit }}=\underbrace{Q S(h, t)}_{\text {flux in }}-\underbrace{Q S(h+\Delta h, t)}_{\text {flux out }} \\
-\underbrace{\int_{h}^{h+\Delta h} A\left[\frac{\mu(S)}{Y}-\left(1-f_{p}\right) b\right] X \mathrm{~d} x}_{\text {consumption per time unit }} .
\end{gathered}
$$

Dividing the previous expression by $A \Delta h$ and taking the limit $\Delta h \rightarrow 0$ we get

$$
\frac{\partial S}{\partial t}+\frac{Q}{A} \frac{\partial S}{\partial h}=-\left[\frac{\mu(S)}{Y}-\left(1-f_{p}\right) b\right] X .
$$

In the same way, for the biomass and the inert matter we get

$$
\begin{aligned}
& \frac{\partial X}{\partial t}+\frac{Q}{A} \frac{\partial X}{\partial h}=[\mu(S)-b] X \\
& \frac{\partial Z}{\partial t}+\frac{Q}{A} \frac{\partial Z}{\partial h}=f_{p} b X
\end{aligned}
$$

In steady-state conditions, (12)-(14) become

$$
\begin{aligned}
& \frac{Q}{A} \frac{\partial S}{\partial h}=-\left[\frac{\mu(S)}{Y}-\left(1-f_{p}\right) b\right] X, \\
& \frac{Q}{A} \frac{\partial X}{\partial h}=[\mu(S)-b] X, \\
& \frac{Q}{A} \frac{\partial Z}{\partial h}=f_{p} b X,
\end{aligned}
$$

where $S=S(h), X=X(h)$ and $Z=Z(h)$ are the steadystate concentrations along the PFR that should satisfy the boundary conditions: $S(0)=S_{1}, X(0)=X_{1}$ and $Z(0)=Z_{1}$. The effluent concentrations are $S_{e}=S\left(h_{\max }\right)$, $X_{e}=X\left(h_{\max }\right)$ and $Z_{e}=Z\left(h_{\max }\right)$, where $h_{\max }$ refers to the maximum length of the PFR.

See in (12) that when $b=0$, the partial derivative for $S$ is negative, i.e. the substrate decreases as long as the PFR length increases. When $b>0$, there is a condition for (12) to be positive, which means that there is a minimum effluent substrate concentration $S_{e}^{\min }$ achievable in the effluent of the PFR, and is given by

$$
S_{e}^{\min }=\frac{K_{\mathrm{S}} Y b\left(1-f_{p}\right)}{\mu_{\max }-Y b\left(1-f_{p}\right)} .
$$

\subsection{Optimization problems}

Given $N$ CSTRs in series as shown in Figure 1, assume that the process is in steady-state condition. It is of interest to get the solution of the following optimization problems:

Problem 1N. Given a total volume $V_{\text {tot }}$ of $N$ CSTRs in series, find the optimal volume distribution that minimize the effluent substrate concentration $S_{N}$ expressed in (6), i.e.,

$$
\underset{\left(V_{1}, \ldots, V_{N}\right)}{\operatorname{minimize}}\left\{S_{N}\left(V_{1}, \ldots, V_{N}\right)\right\}
$$

subject to

$$
V_{1}>V_{1}^{\min },\left\{V_{i}\right\}_{i=2}^{N}>0, \sum_{i=1}^{N} V_{i}=V_{\text {tot }}
$$

Problem 2N. Given an effluent substrate concentration $S_{e}<S_{\text {in }}$ of $N$ CSTRs in series, find the optimal volume distribution that minimize the total volume $V_{\text {tot }}$, i.e.,

$$
\underset{\left(V_{1}, \ldots, V_{N}\right)}{\operatorname{minimize}}\left\{V_{\mathrm{tot}}=\sum_{i=1}^{N} V_{i}\right\}
$$


subject to

$$
V_{1}>V_{1}^{\min },\left\{V_{i}\right\}_{i=2}^{N}>0, S_{N}\left(V_{1}, \ldots, V_{N}\right)=S_{e}
$$

For a large number of $N$ CSTRs in series, an alternative to solve Problem $1 \mathrm{~N}$ and Problem $2 \mathrm{~N}$ is by considering the volumes $V_{2}, \ldots, V_{N}$ as a PFR. This gives two optimization problems to solve:

Problem 1PFR. Given a total volume $V_{\text {tot }}>V_{1}^{\text {min }}$ of a process formed by a CSTR followed by a PFR, find the optimal volume $V_{1}$ of the CSTR that minimizes the effluent substrate concentration $S_{e}$ of the PFR, i.e.,

$$
\underset{\left(V_{1}\right)}{\operatorname{minimize}}\left\{S_{e}\left(V_{1}\right)\right\},
$$

subject to

$$
V_{1}^{\min }<V_{1} \leq V_{\text {tot }}
$$

Problem 2PFR. Given an effluent substrate concentration $S_{e}<S_{\text {in }}$ of a CSTR followed by a PFR, find the optimal volumes $V_{1}$ of the CSTR and $V_{\mathrm{PFR}}$ of the PFR that minimize the total volume $V_{\text {tot }}=V_{1}+V_{\mathrm{PFR}}$, i.e.,

$$
\underset{\left(V_{1}, V_{\mathrm{PFR}}\right)}{\operatorname{minimize}}\left\{V_{\mathrm{tot}}=V_{1}+V_{\mathrm{PFR}}\right\}
$$

subject to

$$
S_{\mathrm{PFR}}=S_{e},
$$

where $S_{\mathrm{PFR}}$ is the effluent substrate concentration of the PFR.

\section{Numerical illustration}

This section shows a numerical example of the process described in the previous section. Let assume the following parameter values: $V_{\text {tot }}=1.1 \mathrm{~m}^{3}, Q=1 \mathrm{~m}^{3} / \mathrm{d}$, $A=0.428 \mathrm{~m}^{2}, \mu_{\max }=2 \mathrm{~d}^{-1}, b=[0,0.8] \mathrm{d}^{-1}, f_{p}=[0,1]$, $Y=0.8, K_{\mathrm{S}}=1.2 \mathrm{~kg} / \mathrm{m}^{3}, S_{\text {in }}=10 \mathrm{~kg} / \mathrm{m}^{3}$. The simulations were performed using the software Matlab.

\subsection{Response of the system for a given $V_{1}$}

Given a total volume $V_{\text {tot }}$ and the volume of the first CSTR, we show the value of the substrate, biomass and inert concentrations for the remaining volume $\left(V_{\text {tot }}-V_{1}\right)$ for some values of $N$ CSTRs in series and for the configuration CSTR+PFR. In this case, $V_{1}$ should be larger than $V_{1}^{\text {min }}$ (cf. (10)). See that $V_{1}^{\mathrm{min}}$ depends on $b$. As illustration, this example shows results for $b=0$ and $b=0.1$. Hence, to use the same $V_{1}$ in all the evaluations, we take the most restrictive case, i.e. $b=0.1$, giving $V_{1}^{\mathrm{min}}=0.593$. We select $V_{1}=1.2 V_{1}^{\mathrm{min}}=0.712$. Results are shown in Figure 3 for three different combinations of $b$ and $f_{p}$.

See that, as expected, the biomass concentration decreases and the substrate concentration increases when $b$ increases. Also see that the inert concentration increases as $f_{p}$ increases.
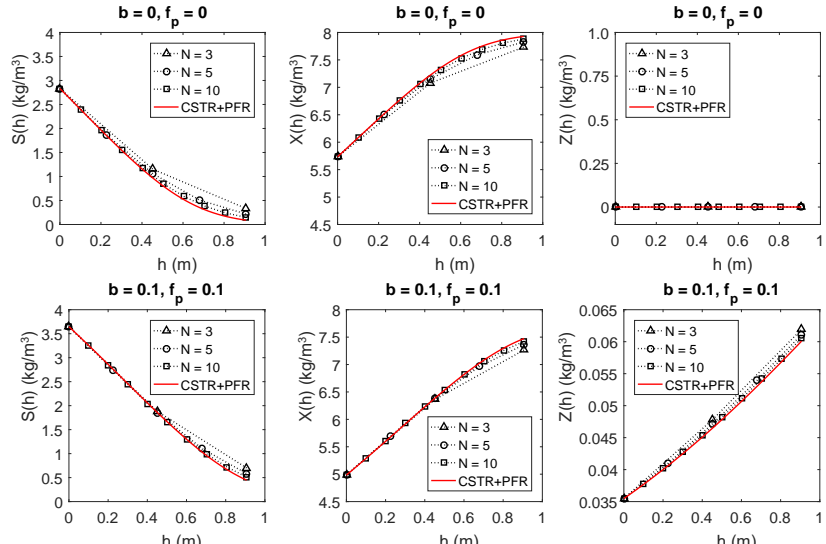

$b=0.1, f_{p}=0.1$
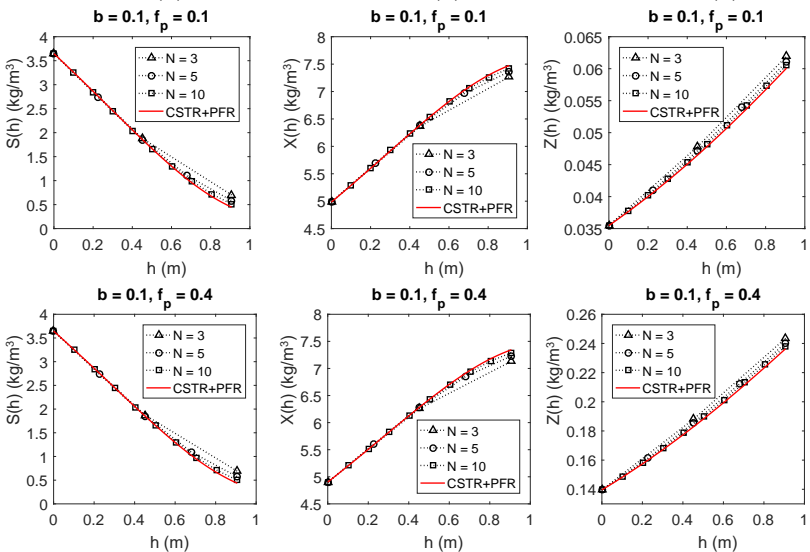

Figure 3. Response of $S, X$ and $Z$ for a given $V_{1}$. Results for $N$ CSTRs and CSTR+PFR. Top: $b=f_{p}=0$. Middle: $b=0.1, f_{p}=$ 0.1 . Bottom: $b=0.1, f_{p}=0.4$.

\subsection{Optimal design for a given total volume}

In this example, the aim is to find the solution of Problem $1 \mathrm{~N}$ and Problem 1PFR. In order to show the optimal design in an illustrative way, we proceed to get the effluent substrate concentration $S_{e}$ for different values of $V_{1}$.

For the case of Problem $1 \mathrm{~N}$, the volumes $V_{2}$ to $V_{N}$ are optimized using the Matlab function fmincon since it can find the minimum value of a multivariate function with linear and nonlinear constraints. See that Problem 1N only has linear constraints.

For the case of Problem 1PFR, $S_{e}$ is obtained by solving (15)-(17). After the evaluation with the full possible range of $V_{1}$, the optimum $V_{1}$ that gives the minimum effluent concentration is obtained. The results are shown in Figure 4 for the case of $N=2,3,5,10$ and for the case of CSTR+PFR, for some values of $b$ and $f_{p}$.

See that the solution given by the $N$ CSTRs converges to the solution of CSTR+PFR as $N$ increases. Note that the steady-state solutions converge to the value $S_{e}=S_{\text {in }}=10$ which corresponds to $V_{1}=V_{1}^{\mathrm{min}}$, and to the value $S_{e}=S_{1}$ when $V_{1}=V_{\text {tot }}$, which is the case of a single CSTR.

\subsection{Optimal vs. sub-optimal design for a given total volume}

Given a total volume $V_{\text {tot }}$, two volume designs of $N$ CSTRs are compared: (a) suboptimal design, where volume $V_{1}$ is taken from the optimal design of CSTR+PFR and the rest of the volumes are equally sized; and (b) optimal design, where all the volumes are optimized. The results are shown in Figure 5 for different values in $b$ and $f_{p}$.

As expected, the effluent substrate concentration increases as $b$ increases. Note also that, when $b$ increases, 

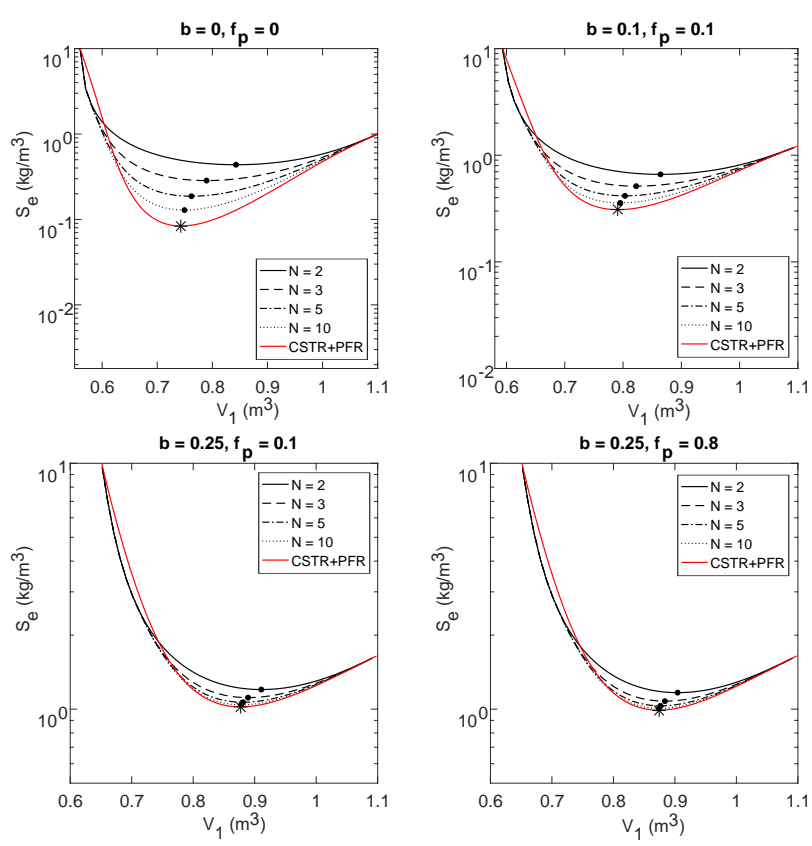

Figure 4. Steady-state solution of the effluent substrate concentration as a function of $V_{1}$. Results shown for $N$ CSTRs and CSTR+PFR, and for different values in $b$ and $f_{p}$. The volume $V_{1}$ that gives the minimum effluent is marked with black dot (for $N$ CSTRs) and with asterisk (for CSTR+PFR).

not much $S_{e}$ reduction is obtained when $N$ increases. This is because the minimum volume to avoid wash-out increases as $b$ increases (see (10)), which makes $V_{1}$ to be closer to $V_{\text {tot }}$.

\subsection{Optimal design for a given $S_{e}$}

In this example, the aim was to compare the solution of Problem 2N and Problem 2PFR. This is, we compare the minimum total volume required in $N$ CSTRs to achieve a given effluent substrate concentration $S_{e}$, with the minimum total volume required in the case of CSTR+PFR. Note that there is no bounds imposed for the total volume required in both problems. Both problems were solved with the Matlab function fmincon. Note that these problems involve linear constraints (regarding the volume distribution) and nonlinear constraints (regarding the effluent substrate concentration).

To facilitate the comparison, the ratio $V_{\mathrm{opt}} / V(N)$ with respect to the ratio $S_{e} / S_{\text {in }}$ is plotted, where $V_{\mathrm{opt}}=V_{1}^{*}+A h^{*}$, and $V(N)$ refers to the minimum total volume required for $N$ CSTRs. We evaluate this example for up to $N=5$ and for $S_{e} / S_{\text {in }}$ between $10^{-2}$ and 1 . Results are shown in Figure 6 for some values in $b$ and $f_{p}$, the value $S_{e}^{\min } / S_{\text {in }}$ (cf. (18)) is also shown.

See that, for a given effluent requirement, the ratio between $V_{\text {opt }}$ and $V(N)$ approaches to one as $N$ increases, i.e. the solution given by Problem $2 \mathrm{~N}$ converges to the solution given by Problem 2PFR when more CSTRs are involved in the process. See also that when the effluent requirement is less exigent $\left(S_{e} / S_{\text {in }}\right.$ close to $\left.10^{-1}\right)$, the total volume of both problems is very similar (i.e. ratio very
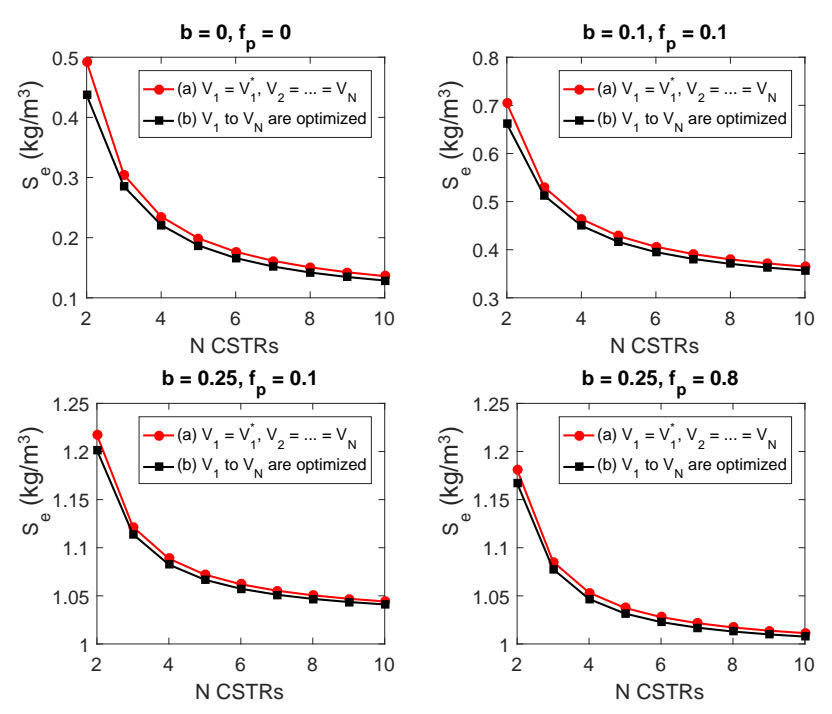

Figure 5. Effluent substrate concentration as a function of $N$ CSTRs for two configurations. $V_{1}^{*}$ refers to the optimal volume of the case CSTR+PFR. Results shown for different values in $b$ and $f_{p}$.

\section{close to one).}

Note that the ratio $V_{\text {opt }} / V(N)$ decreases as $b$ increases, and that this decrease is more evident for low $N$. Since increasing $b$ promotes that more biomass die (which later will become part as substrate and the remaining as inert), then a larger total volume is needed to reduce the substrate concentration to the required level. Hence, compared to the case CSTR+PFR, a larger total volume is needed for low $N$ CSTRs.

On the other hand, the ratio $V_{\text {opt }} / V(N)$ increases as $f_{p}$ increases. Since increasing $f_{p}$ promotes more inert and less substrate concentration, then it has the opposite effect in the ratio of volumes compared to increasing $b$.

\section{Conclusions}

A process consisting of $N$ CSTRs in series has been studied in steady-state conditions. A simple model for the biological reactions based on ordinary differential equations has been used, which describes the growth of biomass via consuming dissolved substrate and producing new dissolved substrate and inert particulate matter.

Numerical results suggest that it is possible to find an optimal distribution of CSTR volumes in order to either: (i) minimize the effluent substrate concentration for a given volume, or (ii) minimize the total volume to fulfill an imposed effluent substrate concentration. The configuration of CSTRs in series was compared to a configuration formed by one CSTR followed by a PFR, i.e. CSTR+PFR. In the configuration CSTR+PFR, an optimal distribution of volume was also found. This configuration can be used as an approximation to the optimal design, by finding the optimal design of the first CSTR and then dividing the remaining volume in equally distributed CSTRs.

The decay rate $b$ has a strong influence in the optimiza- 

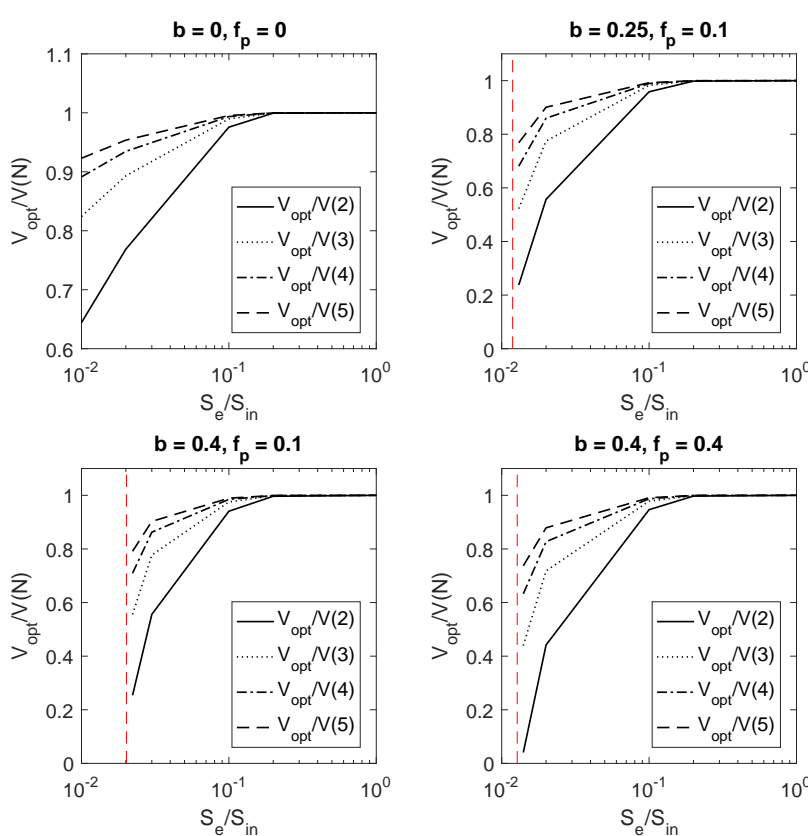

Figure 6. Minimum total volume needed for a given effluent substrate concentration $S_{e} . V_{\text {opt }}$ refers to the solution of Problem 2PFR, and $V(N)$ refers to the solution of Problem 2N. Results shown for different values in $b$ and $f_{p} . S_{e}^{\min } / S_{\text {in }}$ is indicated with a vertical red dashed line.

tion results of both the $N$ CSTRs and the CSTR+PFR. The amount of substrate generated by increasing $b$ makes it less determinant to include a PFR in the process.

This study assumed the hydrolysis as an instantaneous step, since part of the product generated by the biomass is considered already dissolved. A possible extension of this work is to include a hydrolysis step, which involves adding a particulate substrate concentration in the process modelling.

\section{References}

I. M. Abu-Reesh. Optimal design for CSTR's in series using reversible Michaelis-Menten reactions. Bioprocess Engineering, 15(5):257-264, 1996. doi:10.1007/bf02391587.

A. Braha and F. Hafner. Use of monod kinetics on multistage bioreactors. Water Research, 19(10):1217-1227, 1985. doi:10.1016/0043-1354(85)90174-5.

D. E. Contois. Kinetics of bacterial growth: Relationship between population density and specific growth rate of continuous cultures. Journal of General Microbiology, 21(1-3): 40-50, 1959.

C. D. de Gooijer, W. A.M. Bakker, H. H. Beeftink, and J. Tramper. Bioreactors in series: An overview of design procedures and practical applications. Enzyme and Microbial Technology, 18(3):202-219, 1996. doi:10.1016/01410229(95)00090-9.

S. Diehl, J. Zambrano, and B. Carlsson. Steady-state analysis of activated sludge processes with a settler model including sludge compression. Water Research, 88:104-116, 2016. doi:10.1016/j.watres.2015.09.052.
S. Diehl, J. Zambrano, and B. Carlsson. Steady-state analyses of activated sludge processes with plug-flow reactor. Journal of Environmental Chemical Engineering, 5(1):795-809, 2017. doi:10.1016/j.jece.2016.06.038.

C. A. Gómez-Pérez and J. Espinosa. The design analysis of continuous bioreactors in series with recirculation using Singular Value Decomposition. Chemical Engineering Research and Design, 125:108-118, 2017. doi:10.1016/j.cherd.2017.06.030.

C. P. L. Grady Jr., G. T. Daigger, and H. C. Lim. Biological Wastewater Treatment. Marcel Dekker, Inc., New York and Basel, 1999. ISBN 0-8247-8919-9.

G. A. Hill and C. W. Robinson. Minimum tank volumes for CFST bioreactors in series. The Canadian Journal of Chemical Engineering, 67(5):818-824, 1989. doi:10.1002/cjce.5450670513.

K. Ch. A. M. Luyben and J. Tramper. Optimal design for continuous stirred tank reactors in series using Michaelis-Menten kinetics. Biotechnology and Bioengineering, 24(5):12171220, 1982. doi:10.1002/bit.260240518.

J. Monod. The growth of bacterial cultures. Annual Review of Microbiology, 3(1):371-394, 1949.

M. I. Nelson and A. Holder. A fundamental analysis of continuous flow bioreactor models governed by Contois kinetics. II. Reactor cascades. Chemical Engineering Journal, 149(1-3): 406-416, 2009. doi:10.1016/j.cej.2009.01.028.

J. Zambrano, B. Carlsson, and S. Diehl. Optimal steady-state design of zone volumes of bioreactors with Monod growth kinetics. Biochemical Engineering Journal, 100:59-66, 2015. doi:10.1016/j.bej.2015.04.002. 